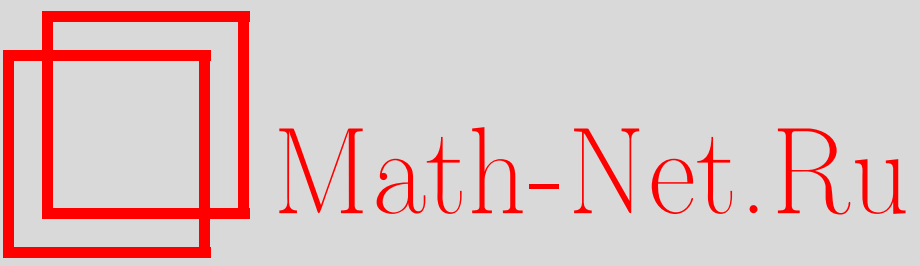

Г. М. Жиислин, Спектральные свойства гамильтонианов заряженных систем в однородном магнитном поле. II. О структуре чисто точечного спектра, $T M \Phi$, 2003, том 134, номер 2, 273-288

DOI: https://doi.org/10.4213/tmf157

Использование Общероссийского математического портала Math-Net.Ru подразумевает, что вы прочитали и согласны с пользовательским соглашением

http://www.mathnet.ru/rus/agreement

Параметры загрузки:

IP : 54.210 .77 .194

26 апреля 2023 г., 09:04:44 


\section{СПЕКТРАЛЬНЫЕ СВОЙСТВА ГАМИЛЬТОНИАНОВ ЗАРЯЖКННЫХ СИСТЕМ В ОДНОРОДНОМ МАГНИТНОМ ПОЛЕ. ІІ. О СТРУКТУРЕ ЧИСТО ТОЧЕЧНОГО СПЕКТРА}

На основе результатов предыдущей работы автора исследуется чисто точечный спектр операторов энергии $H\left(P_{\Sigma}\right)$ многочастичных заряженных квантовых систем в однородном магнитном поле при фиксации суммы $P_{\Sigma}$ компонент псевдомомента системы. Доказано, что для систем с короткодействием при некоторых условиях (выполняющихся, например, для системы двух разноименно заряженных частиц) дискретный спектр $H\left(P_{\Sigma}\right)$ бесконечен даже в случае финитных потенциалов; для систем с дальнодействием типа $(+)$-ионов любых атомов (включая и сами ионы) дискретный спектр бесконечен.

Ключевые слова: гамильтониан, однородное магнитное поле, спектральные свойства, относительное движение, псевдомомент.

\section{1. ВВЕДЕНИЕ}

Настояшая работа завершает начатое в статье [1] исследование спектра гамильтонианов $H$ заряженных многочастичных систем $Z_{1}=\{1,2, \ldots, n\}$ в однородном магнитном поле. В [1] была установлена обшая характеристика спектра и найдено положение сплошного спектра как при фиксированном значении суммы $P_{\Sigma}=P_{x}+P_{y}$ компонент псевдомомента, так и без такой фиксации ${ }^{1)}$. В данной работе мы изучаем структуру чисто точечного спектра рассматриваемых гамильтонианов.

Основные результаты статьи [1] при фиксации $P_{\Sigma}$ (или без нее) таковы.

1. Для систем с короткодействием доказано, что если граница $\mu$ сплошного спектра $Z_{1}$ определяется распадением $Z_{2}=\left(C_{1}, C_{2}\right)$ системы $Z_{1}$ на два устойчивых кластера $C_{j}$ и потенциалы взаимодействия $V_{i j}$ частиц из разных кластеров неположительны и не все равны нулю, то дискретный (или чисто точечный бесконечно вырожденный) спектр

\footnotetext{
1) Фиксация $P_{\Sigma}$ заменяет невозможное в общем случае отделение движения центра масс системы в плоскости $x, y[2]$.
}

* Научно-исследовательский радиофизический институт, Н. Новгород, Россия. E-mail: greg@nirfi.sci-nnov.ru 
бесконечен даже при финитных потенциалах $V_{i j}$. Установлено, что причиной этого является бесконечное вырождение числа $\mu$ как собственного значения гамильтониана составной системы $Z_{2}$. Показано, что вышеупомянутые условия выполнены для системы двух частиц с зарядами $e_{1}$ и $e_{2}$, если $e_{1}+e_{2} \neq 0, e_{1} e_{2}<0$.

2. Для систем с длиннодействием типа $(+)$-ионов любых атомов (включая и сами ионы) доказана бесконечность дискретного (или чисто точечного бесконечно вырожденного) спектра.

Мы будем рассматривать только случай фиксированного значения $P_{\Sigma}$, после чего результаты без фиксации $P_{\Sigma}$ получаются автоматически применением теоремы 2.1 из работы [1]. Доказательство утверждений 1 и 2 при фиксированном $P_{\Sigma}$ состоит в построении для всех натуральных $m$ таких подпространств $L_{m}$ размерности $m$, что на любой нормированной функции $\psi \in L_{m}$ выполняется $(H \psi, \psi)<\mu$. Данное построение основано на том, что граница $\mu$ сплошного спектра $H$ является собственным значением оператора энергии распавшейся системы. Для систем с короткодействием этот факт при $n>2$ постулируется, для случая длиннодействия мы его доказываем. Именно в этом доказательстве преодолеваются главные трудности работы, ибо оно приводит к необходимости рассмотрения цепочки операторов, отвечающих составным системам и получаемых из $Z_{1}$ последовательным удалением “электронов", причем для каждого из таких операторов требовалось найти положение сплошного спектра (в работе [1] такие операторы не изучались, за исключением первого оператора цепочки) и доказать, что нижняя граница каждого из них есть собственное значение.

Ранее какие-либо результаты о дискретном и чисто точечном спектре гамильтонианов заряженных систем в однородном магнитном поле отсутствовали как при фиксации какой-либо компоненты псевдомомента или суммы компонент $P_{\Sigma}$, так и без нее. Часть утверждений работы была анонсирована в статье [3].

\section{2. ОПРЕДЕЛЕНИЯ И ОСНОВНЫЕ РЕЗУЛЬТАТЫ}

2.1. Рассмотрим квантовую систему $Z_{1}=\{1,2, \ldots, n\}$ из $n$ частиц в однородном магнитном поле $\mathcal{F} \in(0 \div 0.2 B)$, где $B>0$ - произвольная константа. Пусть $m_{i}, e_{i}, r_{i}=$ $\left(x_{i}, y_{i}, z_{i}\right)$ - масса, заряд и радиус-вектор $i$-й частицы, $r_{s t}=r_{s}-r_{t}, V_{s t}\left(r_{s t}\right)$ - потенциал взаимодействия частиц $s$ и $t$ между собой. Будем считать всюду, что

$$
\begin{gathered}
V_{s t}\left(-r_{1}\right)=V_{s t}\left(r_{1}\right)=\bar{V}_{s t}\left(r_{1}\right) ; \quad V_{s t}\left(r_{1}\right) \in \mathcal{L}_{2, l o c}\left(R^{3}\right) ; \\
V_{s t}\left(r_{1}\right) \rightarrow 0 \text { при } \quad\left|r_{1}\right| \rightarrow \infty .
\end{gathered}
$$

Пусть $M=\sum_{j=1}^{n} m_{j}, r_{\mathrm{c}}=\left(\alpha_{01}, \alpha_{02}, \alpha_{03}\right)=\sum_{j=1}^{n} m_{j} r_{j} M^{-1}$. Если ввести в гамильтониане $\mathcal{H}$ системы $Z_{1}$ [1] относительные координаты $q_{j}=\left(q_{j 1}, q_{j 2}, q_{j 3}\right)=r_{j}-r_{\mathrm{c}}$, координаты $\xi_{0}=(1 / 2)\left(\alpha_{01}-\alpha_{02}\right), \eta_{0}=(1 / 2)\left(\alpha_{01}+\alpha_{02}\right), \zeta_{0}=\alpha_{03}$ и отделить движение центра масс $Z_{1}$ в направлении поля, то получим оператор

$$
\mathcal{H}_{0}=T_{\perp}+T_{03}+V(q)
$$


где

$$
\begin{gathered}
T_{\perp}=\sum_{j=1}^{n} \sum_{p=1}^{2} m_{j}^{-1}\left\{B_{j p}+\frac{m_{j}}{2 M i}\left[\frac{\partial}{\partial \eta_{0}}-(-1)^{p} \frac{\partial}{\partial \xi_{0}}\right]+\right. \\
\left.+(-1)^{p+1} B e_{j} q_{j \bar{p}}+B e_{j}\left[(-1)^{p+1} \eta_{0}-\xi_{0}\right]\right\}^{2}, \\
T_{03}=\sum_{j=1}^{n} m_{j}^{-1} B_{j 3}^{2}, \quad B_{j p}=\frac{1}{i} \sum_{s=1}^{n} \frac{\partial}{\partial q_{s p}}\left(\delta_{s j}-m_{j} M^{-1}\right), \\
\bar{p}=1 \quad \bar{p}=2 \text { при } p=1 ; \\
V(q)=\frac{1}{2} \sum_{s, t=1, s \neq t}^{n} V_{s t}\left(q_{s}-q_{t}\right), \quad q=\left(q_{1}, \ldots, q_{n}\right) .
\end{gathered}
$$

Оператор $\mathcal{H}_{0}$ рассматривается в пространстве $\bar{R}_{0}^{(3 n-1)}=\left\{q, \xi_{0}, \eta_{0}\right\}$. Согласно работе [1] $\mathcal{H}_{0}$ унитарно эквивалентен оператору

$$
\mathcal{H}_{0}\left(\lambda_{0}\right)=T_{\perp}\left(\lambda_{0}\right)+T_{03}+V(q)
$$

где

$$
\begin{gathered}
T_{\perp}\left(\lambda_{0}\right)=\sum_{j=1}^{n} \sum_{p=1}^{2} \frac{1}{m_{j}}\left(A_{j p}^{(0)}+h_{j p 0}+\frac{m_{j}}{2 M} \lambda_{0}\right)^{2}, \quad h_{j p 0}=\frac{m_{j}}{2 M i}(-1)^{p+1} \frac{\partial}{\partial \xi_{0}}-F_{j} \xi_{0}, \\
A_{j p}^{(0)}=B_{j p}-m_{j}(2 M)^{-1} D_{-}-(-1)^{p} B e_{j} q_{j \bar{p}}, \quad D_{ \pm}=D_{ \pm}(q)=B \sum_{j=1}^{n} e_{j}\left(q_{j 1} \pm q_{j 2}\right), \\
F_{j}=B Q m_{j} M^{-1}+B e_{j} .
\end{gathered}
$$

Оператор $\mathcal{H}_{0}\left(\lambda_{0}\right)$ будет рассматриваться как в пространстве $R_{0}^{(3 n-1)}=\left\{q, \xi_{0}, \lambda_{0}\right\}$, так и в $R_{0}^{(3 n-2)}=\left\{q, \xi_{0}\right\}$ при фиксированном $\lambda_{0}$. В последнем случае вместо $\mathcal{H}_{0}\left(\lambda_{0}\right)$ мы будем писать $\widehat{\mathcal{H}}_{0}\left(\lambda_{0}\right)$. Операторы $\mathcal{H}_{0}, \mathcal{H}_{0}\left(\lambda_{0}\right)$ и $\widehat{\mathcal{H}}_{0}\left(\lambda_{0}\right)$, определенные на $C_{0}^{2}$, полуограничены снизу соответственно в $\mathcal{L}_{2}\left(\bar{R}_{0}^{(3 n-1)}\right), \mathcal{L}_{2}\left(R_{0}^{(3 n-1)}\right)$ и $\mathcal{L}_{2}\left(R_{0}^{(3 n-2)}\right)$. Расширим их до самосопряженных, сохраняя прежние обозначения. Для произвольного оператоpa $L$ обозначим через $\sigma_{\mathrm{p}}(L), \sigma_{\mathrm{pp}}(L), \sigma_{\mathrm{d}}(L), \sigma_{\mathrm{ess}}(L)$ и $\sigma(L)$ соответственно точечный, чисто точечный, дискретный, существенный и полный спектры оператора $L$. Пусть $H=\widehat{\mathcal{H}}_{0}(0)$. Согласно теореме 2.1 работы [1] $\sigma\left(\widehat{\mathcal{H}}_{0}\left(\lambda_{0}\right)\right)$ не зависит от $\lambda_{0}$ и $\sigma\left(\mathcal{H}_{0}\left(\lambda_{0}\right)\right)=$ $\sigma(H)$, но каждая точка $\nu$ из $\sigma\left(\mathcal{H}_{0}\left(\lambda_{0}\right)\right)$ имеет дополнительное бесконечное вырождение по сравнению с этой же точкой в $\sigma(H)$. Поэтому далее мы будем изучать спектр оператора $H$.

2.2. Пусть $Z_{k}=\left(C_{1}, \ldots, C_{k}\right)$ - разбиение $Z_{1}$ на $k, 2 \leqslant k \leqslant n$, не взаимодействующих между собой кластеров $C_{j}, Z_{1}=\bigcup_{j=1}^{k} C_{j}, C_{j} \cap C_{i}=\varnothing, j \neq i$. Положим

$$
H\left(Z_{k}\right)=H-\frac{1}{2} \sum_{i, j=1, i \neq j}^{k} \sum_{s \in C_{i}, t \in C_{j}} V_{s t}\left(q_{s}-q_{t}\right) .
$$


Далее считаем $Z_{k}=Z_{2}=\left(C_{1}, C_{2}\right)$ и положим $M_{j}=\sum_{s \in C_{j}} m_{s}, Q_{j}=\sum_{s \in C_{j}} e_{s}, \alpha_{j}=$ $\left(\alpha_{j 1}, \alpha_{j 2}, \alpha_{j 3}\right)=\sum_{s \in C_{j}} m_{s} q_{s} M_{j}^{-1}$. Введем в каждом кластере $C_{j}$ относительные координаты $q_{s}^{(1)}=q_{s}-\alpha_{j}$ при $s \in C_{j}$ и, кроме того, координаты

$$
\xi_{1}=\frac{1}{2}\left(\alpha_{11}-\alpha_{12}\right), \quad \eta_{1}=\frac{1}{2}\left(\alpha_{11}+\alpha_{12}\right), \quad \zeta_{1}=\alpha_{13}-\alpha_{23} .
$$

Тогда в новых координатах оператор $H\left(Z_{2}\right)$ запишется в виде [1]

$$
H\left(Z_{2}\right)=H_{0}\left(Z_{2}\right)-M M_{1}^{-1} M_{2}^{-1} \frac{d^{2}}{d \zeta_{1}^{2}}
$$

где

$$
\begin{gathered}
H_{0}\left(Z_{2}\right)=\sum_{k=1}^{2}\left(T_{\perp}\left[C_{k}\right]+T_{03}\left[C_{k}\right]+V\left[C_{k}\right]\right), \\
T_{\perp}\left[C_{k}\right]=\sum_{j \in C_{k}} \sum_{p=1}^{2} \frac{1}{m_{j}}\left\{\tilde{A}_{j p}(k)+h_{j p 0}-(-1)^{k} \frac{m_{j} M_{2}}{2 i M M_{k}}\left((-1)^{p+1} \frac{\partial}{\partial \xi_{1}}+\frac{\partial}{\partial \eta_{1}}\right)+\right. \\
\left.+B e_{j}(-1)^{k} M_{1} M_{k}^{-1}\left(\xi_{1}+(-1)^{p} \eta_{1}\right)-m_{j} M^{-1} M_{2}^{-1}\left(Q_{1} M_{2}-Q_{2} M_{1}\right) \xi_{1} B\right\}^{2}, \\
T_{03}\left[C_{k}\right]=\sum_{j \in C_{k}} m_{j}^{-1} B_{j 3}^{2}(k), \quad V\left[C_{k}\right]=\frac{1}{2} \sum_{s, t \in C_{k}, s \neq t} V_{s t}\left(q_{s}^{(1)}-q_{t}^{(1)}\right), \\
\tilde{A}_{j p}(k)=B_{j p}(k)-m_{j}(2 M)^{-1} D_{-}\left(q^{(1)}\right)-(-1)^{p} B e_{j} q_{j \bar{p}}^{(1)}, \quad p=1,2, \\
B_{j p}(k)=\frac{1}{i} \sum_{s \in C_{k}} \frac{\partial}{\partial q_{s p}^{(1)}}\left(\delta_{s j}-m_{j} M_{k}^{-1}\right) .
\end{gathered}
$$

Оператор $H_{0}\left(Z_{2}\right)$ определен в пространстве $\bar{R}_{0}^{(3 n-3)}=\left\{q^{(1)}, \xi_{0}, \xi_{1}, \eta_{1}\right\}$. Наряду с ним рассмотрим действуюший в пространстве $R_{0}^{(3 n-3)}=\left\{q^{(1)}, \xi_{0}, \xi_{1}, \lambda_{1}\right\}$ оператор

$$
H_{0}\left(Z_{2}, \lambda_{1}\right)=\sum_{k=1}^{2}\left(T\left[C_{k}, \lambda_{1}\right]+V\left[C_{k}\right]\right)
$$

где

$$
\begin{gathered}
T\left[C_{k}, \lambda_{1}\right]=T_{\perp}\left[C_{k}, \lambda_{1}\right]+T_{03}\left[C_{k}\right], \\
T_{\perp}\left[C_{k}, \lambda_{1}\right]=\sum_{j \in C_{k}} \sum_{p=1}^{2} m_{j}^{-1}\left\{A_{j p}(k)+\sum_{s=0}^{1} h_{j p s}(k)+(-1)^{k+1} m_{j} M_{2}\left(2 M_{k} M\right)^{-1} \lambda_{1}\right\}^{2}, \\
A_{j p}(k)=B_{j p}(k)-m_{j}\left(2 M_{k}\right)^{-1} D_{-}\left(q^{(1)}, k\right)+(-1)^{p+1} e_{j} q_{j \bar{p}}^{(1)} B, \\
D_{-}\left(q^{(1)}, k\right)=B \sum_{t \in C_{k}}\left(q_{t 1}^{(1)}-q_{t 2}^{(1)}\right), \quad h_{j p 0}(k)=(-1)^{p+1} \frac{1}{i} \frac{m_{j}}{2 M} \frac{\partial}{\partial \xi_{0}}-F_{j}(k) \xi_{0}, \\
h_{j p 1}(k)=(-1)^{k} \frac{M_{1}}{M_{k}}\left(\frac{1}{2 i} \frac{M_{2} m_{j}}{M_{1} M}(-1)^{p} \frac{\partial}{\partial \xi_{1}}+F_{j}(k) \xi_{1}\right), \\
F_{j}(k)=B e_{j}+Q_{k} M_{k}^{-1} B m_{j},
\end{gathered}
$$


$\lambda_{1}$ - одномерная переменная.

Согласно результатам работы [1] $H_{0}\left(Z_{2}\right)$ унитарно эквивалентен $H_{0}\left(Z_{2}, \lambda_{1}\right)$. Если оператор $H_{0}\left(Z_{2}, \lambda_{1}\right)$ будет рассматриваться в пространстве $R_{0}^{(3 n-4)}=\left\{q^{(1)}, \xi_{0}, \xi_{1}\right\}$ при фиксированном $\lambda_{1}$, то мы обозначим его через $\widehat{H}_{0}\left(Z_{2}, \lambda_{1}\right)$. В силу теоремы 2.3 из работы [1] спектр $\sigma\left(\widehat{H}_{0}\left(Z_{2}, \lambda_{1}\right)\right)$ не зависит от $\lambda_{1}$ и $\sigma\left(H_{0}\left(Z_{2}\right)\right)=\sigma\left(\widehat{H}_{0}\left(Z_{2}, 0\right)\right)$, но спектр $\sigma\left(H_{0}\left(Z_{2}\right)\right)$ имеет дополнительное бесконечное вырождение по сравнению со спектром $\sigma\left(\widehat{H}_{0}\left(Z_{2}, 0\right)\right)$ и, в частности, любая точка $\nu \in \sigma_{\mathrm{p}}\left(\widehat{H}_{0}\left(Z_{2}, 0\right)\right)$ есть бесконечно вырожденное собственное значение оператора $H_{0}\left(Z_{2}\right)$. Пусть

$$
\mu:=\min _{Z_{2}} \inf H_{0}\left(Z_{2}\right) \equiv \min _{Z_{2}} \inf \widehat{H}_{0}\left(Z_{2}, 0\right)
$$

В силу теоремы 2.2 из работы $[1] \sigma_{\mathrm{ess}}\left(\mathcal{H}_{0}\right)=[\mu,+\infty)$.

2.3. Теорема 2.1. Пусть существует такое $Z_{2}=\left(C_{1}, C_{2}\right)$, что

$$
\mu \in \sigma_{\mathrm{p}}\left(\widehat{H}_{0}\left(Z_{2}, 0\right)\right) ; \quad Q_{1} Q_{2} \neq 0 ; \quad V_{s t}\left(r_{1}\right) \leqslant 0 ; \quad V_{s t}\left(r_{1}\right) \not \equiv 0
$$

для любых $s, t, s \in C_{1}, t \in C_{2}$. Тогда дискретный спектр оператора Н бесконечен.

ЗАМЕЧАНИЕ 2.1. Условия (2.7) выполняются, например, для находяшейся в однородном магнитном поле заряженной $(Q \neq 0)$ системы $Z_{1}=\{1,2\}$ двух не нейтральных частиц с потенциалом $V_{12}\left(r_{1}\right) \leqslant 0, V_{12}\left(r_{1}\right) \not \equiv 0$, ибо для этой системы сушествует единственное распадение $Z_{2}=\{(1),(2)\}$ и, значит, оператор $\widehat{H}_{0}\left(Z_{2}, 0\right)$ имеет чисто точечный спектр (совокупность орбит Ландау). Таким образом, для рассматриваемой системы $Z_{1}$ спектр $\sigma_{\mathrm{d}}(H)$ будет бесконечным и при короткодействующем, и даже при финитном потенциале $V_{12}$. Причина этого - в бесконечном вырождении собственного значения $\mu$ оператора $H_{0}\left(Z_{2}\right)$. Те же причины имеют место и в произвольных $n$-частичных системax с $n>2$ при выполнении условий (2.7) и короткодействуюших потеншиалах $V_{s t}\left(r_{1}\right)$, $s \in C_{1}, \quad t \in C_{2}$. В случае же длиннодействующих потенциалов бесконечность спектра $\sigma_{\mathrm{d}}(H)$ в условиях теоремы 2.1 обусловливается не только тем, что собственное подпространство $\mathcal{U}_{\mu}$ оператора $H_{0}\left(Z_{2}\right)$, отвечающее его собственному значению $\mu$, бесконечномерно, но и тем, что каждая функция из $\mathcal{U}_{\mu}$ порождает свою бесконечную серию дискретных собственных значений оператора $H$.

ЗАмЕчАнИЕ 2.2. Вместо условия $V_{s t}\left(r_{1}\right) \leqslant 0$ для справедливости теоремы 2.1 можно потребовать выполнения следуюшего более слабого (но трудного для проверки) условия: в подпространстве $\mathcal{U}_{\mu}$ для любого $N>0$ должно существовать такое подпространство $L_{N}, \operatorname{dim} L_{N} \geqslant N$, что для всех $u \in L_{N}, u \neq \equiv$, выполняется неравенство

$$
\sum_{s \in C_{1}, t \in C_{2}} \int V_{s t}\left(q_{s}-q_{t}\right)|u|^{2} d q^{(1)} d \xi_{0} d \eta_{0} d \zeta_{1}<0 .
$$

ЗАмечаниЕ 2.3. Автор считает, что если $Z_{1}$ не содержит нейтральных подсистем и условие $V_{s t}\left(r_{1}\right) \leqslant 0$ выполняется для всех $s, t$, то для всех $Z_{2}$ выполняется первое из условий $(2.7)$, т.е. спектр $\sigma_{\mathrm{d}}(H)$ бесконечен, однако это пока не доказано. 
2.4. Рассмотрим теперь систему $Z_{1}^{+}=\{1,2, \ldots, n\}$ типа положительного атомарного иона. Пусть

$$
\begin{gathered}
V_{i j}\left(r_{1}\right)=e_{i} e_{j}\left|r_{1}\right|^{-\gamma}, \quad 0<\gamma<\frac{3}{2} \\
e_{j}=e<0, \quad m_{j}=m, \quad j=2,3, \ldots, n, \quad e_{1}>-(n-1) e .
\end{gathered}
$$

ТеОрема 2.2. Дискретный спектр гамильтониана $H$ системы $Z_{1}^{+}$бесконечен.

ЗАМЕчАНИЕ 2.4. При $\gamma=1$ теорема 2.1 из работы [1] и теорема 2.2 обеспечивают бесконечность числа связанных состояний $(+)$-ионов произвольных атомов при любом фиксированном значении суммы компонент псевдомомента.

2.5. Из полученных выше результатов о спектре оператора $H$ и из теорем 2.1 и 2.2 работы [1] вытекает следующее утверждение о спектре оператора $\mathcal{H}_{0}$.

Tеорема 2.3. Спектр $\sigma\left(\mathcal{H}_{0}\right)$ состоит из всех точек луча $[\mu,+\infty)$ и (при ви-полнении условий (2.7) или (2.9)) из бесконечного числа бесконечно вырожденных собственных значений, накапливающихся $к$ числу $\mu$ слева.

\section{3. ДОКАЗАТЕЛЬСТВА ТЕОРЕМ 2.1 И 2.2}

3.1. Докажем сначала теорему 2.1. Раз $\mu \in \sigma_{\mathrm{p}}\left(\widehat{H}_{0}\left(Z_{2}, 0\right)\right)$, то в силу теоремы 2.3 из работы [1] $\mu$ - бесконечно вырожденное собственное значение оператора $H_{0}\left(Z_{2}\right)$. Пусть $\mathcal{U}_{\mu}$ - отвечаюшее $\mu$ бесконечномерное собственное подпространство оператора $H_{0}\left(Z_{2}\right)$; $u_{j}=u_{j}\left(q^{(1)}, \xi_{0}, \xi_{1}, \eta_{1}\right), j=1,2, \ldots,-$ бесконечная система ортонормированных функций из $\mathcal{U}_{\mu}, I_{Z_{2}}=I_{Z_{2}}(q)=V(q)-V\left[C_{1}\right]-V\left[C_{2}\right]$. Рассмотрим функционал

$$
J[d]=\int\left|\sum_{i=1}^{m} d_{i} u_{i}\right|^{2} I_{Z_{2}}(q) d \Omega,
$$

где $d=\left(d_{1}, \ldots, d_{m}\right), d_{i}$ - любые числа, $|d|^{2}=\sum_{i=1}^{m}\left|d_{i}\right|^{2}=1, d \Omega=d q^{(1)} d \xi_{0} d \xi_{1} d \eta_{1} d \zeta_{1}$, $m>0$ - произвольное натуральное число. При выполнении третьего из условий (2.7) верно, что

$$
2 \alpha:=\max _{d,|d|=1} J_{0}[d]<0 .
$$

Пусть $f_{k}(\tau)=1$ при $|\tau| \leqslant k, f_{k}(\tau)=0$ при $|\tau| \geqslant 2 k$,

$$
f_{k}(\tau)=1-3(\tau-k)^{2} k^{-2}+2(\tau-k)^{3} k^{-3}
$$

при $k \leqslant \tau \leqslant 2 k, f_{k}=f_{k}\left(\zeta_{1}\right), f_{m, k}=\left(\sum_{i=1}^{m} d_{i} u_{i}\right) f_{k}$,

$$
J_{k}[d]=\int\left|f_{m, k}\right|^{2} I_{Z_{2}} d \Omega
$$

Очевидно, что при $|d|=1$ и фиксированном $m$ равномерно по $d$

$$
\lim _{k \rightarrow \infty} J_{k}[d]=J[d] \leqslant 2 \alpha,
$$


поэтому при любых значениях $m$ и $|d|=1$ существует $\bar{k}=\bar{k}(m)$ такое, что при $k>\bar{k}$

$$
J_{k}[d] \leqslant \alpha<0 .
$$

Вычислим величину $L\left[f_{m, k}\right]=\left(H f_{m, k}, f_{m, k}\right)\left\|f_{m, k}\right\|^{-2}$. В силу $(2.4),(2.5)$ и так как $\left\|f_{m, k}\right\|=|d|\left\|f_{k}\right\|=\left\|f_{k}\right\|$, выполняется равенство

$$
L\left[f_{m, k}\right]=\mu+\left(\frac{M}{M_{1} M_{2}}\left\|\frac{d f_{k}}{d \zeta_{1}}\right\|^{2}+\int I_{Z_{2}}(q)\left|f_{m, k}\right|^{2} d \Omega\right)\left\|f_{k}\right\|^{-2} .
$$

Поскольку $\lim _{k \rightarrow \infty}\left\|d f_{k} / d \zeta_{1}\right\|=0$, то сушествует $k_{1}$ такое, что $M\left(M_{1} M_{2}\right)^{-1}\left\|d f_{k} / d \zeta_{1}\right\| \leqslant$ $(1 / 2)|\alpha|$ при $k>k_{1}$. Отсюда и из условия (3.3) следует, что при $k>k_{1}+\bar{k}$

$$
L\left[f_{m, k}\right] \leqslant \mu+\frac{1}{2} \alpha\left\|f_{k}\right\|^{-2}<\mu .
$$

Фиксируем какое-либо $k, k>k_{1}+\bar{k}$. Тогда из (3.5) следует, что на любой нормированной функции $f_{m, k}$ из $m$-мерной линейной оболочки $\mathcal{L}\left\{u_{1} f_{k}, u_{2} f_{k}, \ldots, u_{m} f_{k}\right\}$ квадратичная форма оператора $H$ меньше $\mu$. Значит, размерность линейной оболочки собственных функций дискретного спектра $H$, лежашего слева от $\mu$, не меньше $m$. Так как $m$ произвольно, то теорема 2.1 доказана.

3.2. Доказательство теоремы 2.2 проведем в предположении, что при $Z_{2}=\{(1,2, \ldots$ $\ldots, n-1),(n)\}$

$$
\mu \in \sigma_{\mathrm{p}}\left(H_{0}\left(Z_{2}\right)\right)
$$

а потом докажем, что это предположение верно (п. 3.4 и раздел 4). Заметим, что именно в проверке условия (3.6) заключается основная трудность доказательства теоремы 2.2. Итак, пусть (3.6) верно, $u$ - нормированная собственная функция оператора $H_{0}\left(Z_{2}\right)$, отвечаюшая его собственному значению $\mu, m$ - фиксированное натуральное число, $g_{i 1}\left(\zeta_{1}\right), i=1, \ldots, m,-$ произвольные ортонормированные в $\mathcal{L}_{2}\left(R^{1}\right)$ функции из $C_{0}^{2}, \operatorname{supp} g_{i 1}\left(\zeta_{1}\right) \subseteq[1,2], k>0$ - любое число, $g_{i k}:=g_{i 1}\left(k \zeta_{1}\right) k^{1 / 2}$. Пусть $\mathcal{L}_{k}=\mathcal{L}\left\{u g_{1 k}\right.$, $\left.u g_{2 k}, \ldots, u g_{m k}\right\}$. Очевидно, что функции $g_{i k}, i=1,2, \ldots, m$, ортонормированы и $\operatorname{dim} \mathcal{L}_{k}=m$. Докажем, что при малых $k$ для всех функций $v_{k}$ из $\mathcal{L}_{k},\left\|v_{k}\right\|=1$, вьполняется неравенство

$$
\left(H v_{k}, v_{k}\right)<\mu .
$$

Отсюда, так же как из неравенства (3.5) при доказательстве теоремы 2.1, будет следовать, что спектр $\sigma_{\mathrm{d}}(H)$ бесконечен.

3.3. Так как $v_{k} \in \mathcal{L}_{k}$, то $v_{k}=g_{k} u$, где $g_{k}=\sum_{i=1}^{m} d_{i} g_{i k}, d=\left(d_{1}, \ldots, d_{m}\right)$ - любые числа. Всюду считаем, что $|d|^{2}:=\sum_{i=1}^{m}\left|d_{i}\right|^{2}=1$; тогда $\left\|v_{k}\right\|=\left\|g_{k}\right\|=|d|=1$. В силу $(2.4)$ и $(2.5)$

$$
\left(H v_{k}, v_{k}\right)=\mu+M M_{1}^{-1} M_{2}^{-1}\left\|\frac{d g_{k}}{d \zeta_{1}}\right\|^{2}+L_{k}(d),
$$


где

$$
\begin{aligned}
& L_{k}(d)=\sum_{s=1}^{n-1} e_{s} e J_{s k}(d), \quad J_{s k}(d)=\int\left|v_{k}\right|^{2}\left|P_{s}\right|^{-\gamma} d \Omega, \\
P_{s}= & \left(P_{s 1}, P_{s 2}, P_{s 3}\right)=q_{s}-q_{n}= \\
= & \left(q_{s 1}^{(1)}+m^{-1} M\left(\xi_{1}+\eta_{1}\right), q_{s 2}^{(1)}+m^{-1} M\left(\eta_{1}-\xi_{1}\right), q_{s 3}^{(1)}+\zeta_{1}\right), \\
& d \Omega=d q^{(1)} d \xi_{0} d \xi_{1} d \eta_{1} d \zeta_{1}, \quad d q^{(1)}=d q_{1}^{(1)} \ldots d q_{n-1}^{(1)} .
\end{aligned}
$$

Здесь мы учли формулы $(2.11)$ из работы [1] и тот факт, что $q_{n}^{(1)}=(0,0,0)$. Покажем, что при всех $d,|d|=1$, и малых $k$

$$
L_{k}(d) \leqslant Q_{1} e \beta k^{\gamma}
$$

где $Q_{1}=e_{1}+(n-2) e>0, \beta>0$ и не зависит от $k$ и $d$ при $|d|=1$. Делая замену переменных $\tau=k \zeta_{1}$ в $J_{s k}(d)$ и в $\left\|d g_{k} / d \zeta_{1}\right\|^{2}$, получим

$$
\begin{gathered}
\left\|\frac{d g_{k}}{d \zeta_{1}}\right\|^{2}=k^{2}\left\|\frac{d g_{1}}{d \tau}\right\|^{2}=k^{2} \beta_{0}, \\
J_{s k}(d)=k^{\gamma} \int\left|v_{1}\right|^{2}\left[k^{2}\left(P_{s 1}^{2}+P_{s 2}^{2}\right)+\left(k q_{s 3}^{(1)}+\tau\right)^{2}\right]^{-\gamma / 2} d \Omega_{\tau},
\end{gathered}
$$

где $\beta_{0}$ - константа, не зависящая от $d$ и $k, d \Omega_{\tau}=d q^{(1)} d \xi_{0} d \xi_{1} d \eta_{1} d \tau$. Можно показать, что для любого $\varepsilon>0$ сушествует $k_{0}>0$ такое, что при $k<k_{0}$ и всех $d,|d|=1$, верно

$$
\left.\left|e_{s} e J_{s k}(d) k^{-\gamma}-e_{s} e \int\right| g_{1}(\tau)\right|^{2}|\tau|^{-\gamma} d \tau \mid<\varepsilon .
$$

Поэтому при $k<k_{0}$

$$
L_{k}(d)<k^{\gamma} Q_{1} e \int\left|g_{1}(\tau)\right|^{2}|\tau|^{-\gamma} d \tau+k^{\gamma}(n-1) \varepsilon
$$

Так как функции $g_{i 1}$ линейно независимы, то

$$
2 \beta:=\min _{d,|d|=1} \int\left|\sum_{i=1}^{m} d_{i} g_{i 1}\right|^{2}|\tau|^{-\gamma} d \tau>0
$$

Выбрав в $(3.11) \varepsilon<\beta(n-1)^{-1}$, получаем из этого неравенства выражение (3.9). Подставляя в (3.8) оценки (3.9), (3.10), приходим к неравенству

$$
\left(H v_{k}, v_{k}\right) \leqslant \mu+k^{2} \beta_{0} M M_{1}^{-1} M_{2}^{-1}+Q_{1} e \beta k^{\gamma} .
$$

Так как $Q_{1} e<0, \beta>0,0<\gamma<3 / 2$, то при малых $k$ отсюда следует выражение (3.7). Таким образом, в предположении (3.6) теорема 2.2 доказана. 
3.4. Пусть $Z_{2}=\{(1,2, \ldots, n-1),(n)\}$. Прежде чем доказывать (3.6), заметим, что поскольку для рассматриваемой системы $V_{i j}>0$ при $i, j \geqslant 2$, то для любого разбиения $Z_{2}^{\prime}=\left(C_{1}^{\prime}, C_{2}^{\prime}\right)$ системы $Z_{1}$ выполняется неравенство $\inf H_{0}\left(Z_{2}^{\prime}\right) \geqslant \inf H_{0}\left(Z_{2}\right)$ и, значит, $\mu=\inf H_{0}\left(Z_{2}\right)=\inf \widehat{H}_{0}\left(Z_{2}, 0\right)$. В силу утверждения теоремы 2.3 из работы [1] для доказательства (3.6) достаточно показать, что $\mu \in \sigma_{\mathrm{d}}\left(\widehat{H}_{0}\left(Z_{2}, 0\right)\right)$, для чего надо установить неравенство

$$
\mu<\mu\left(Z_{2}\right)=\inf \left\{\nu \mid \nu \in \sigma_{\text {ess }}\left(\widehat{H}_{0}\left(Z_{2}, 0\right)\right)\right\} .
$$

Для доказательства (3.12) рассмотрим последовательность разбиений $Z_{k+1}=\left(C_{1}^{(k)}\right.$, $\left.C_{2}^{(k)}, \ldots, C_{k+1}^{(k)}\right)$, где $C_{1}^{(k)}=(1,2, \ldots, n-k), C_{s}^{(k)}=(n+s-k-1), s=2, \ldots, k+1$, $k=1,2, \ldots, n-1$. $Z_{k+1}$ - разбиение исходной системы $Z_{1}^{+}$на $(+)$-ион с зарядом $e_{1}+$ $e(n-k+1)$ и не взаимодействуюшие с ним и между собой $k$ электронов с номерами $n+s-k-1, s=2,3, \ldots, k+1$. Введем относительные координаты частиц в кластере $C_{1}^{(k)}$ последовательно для $k=1,2, \ldots, n-1$ :

$$
q_{j}^{(k)}=q_{j}^{(k-1)}-\sum_{s=1}^{n-k} \frac{m_{s} q_{s}^{(k-1)}}{M^{(k)}}, \quad j=1,2, \ldots, n-k,
$$

где $M^{(s)}=M-s m$ - масса частиц кластера $C_{1}^{(s)}, 0 \leqslant s \leqslant n-1, q_{j}^{(0)}=q_{j}$. Сопоставим составной системе $Z_{k+1}$ оператор

$$
H_{0}^{(k)}=T_{0 \perp}^{(k)}+T_{03}^{(k)}+T_{\mathrm{c} \perp}^{(k)}+V^{(k)},
$$

где

$$
\begin{gathered}
T_{0 \perp}^{(k)}=\sum_{j=1}^{n-k} \sum_{p=1}^{2} m_{j}^{-1}\left(A_{j p}^{(k)}-\sum_{s=0}^{k} h_{j p s}^{(k)}\right)^{2}, \quad T_{\mathrm{c} \perp}^{(k)}=\sum_{s=1}^{k} \sum_{p=1}^{2} \ell_{p s}^{2} m^{-1}, \\
T_{03}^{(k)}=\sum_{j=1}^{n-k}\left(B_{j 3}^{(k)}\right)^{2} \frac{1}{m_{j}}, \\
A_{j p}^{(k)}=B_{j p}^{(k)}+(-1)^{p+1} B e_{j} q_{j \bar{p}}^{(k)}-m_{j}\left(2 M^{(k)}\right)^{-1} D_{-}^{(k)}, \\
B_{j p}^{(k)}=\sum_{s=1}^{n-k} \frac{1}{i} \frac{\partial}{\partial q_{s p}^{(k)}}\left(\delta_{j s}-\frac{m_{j}}{M^{(k)}}\right), \\
\ell_{p s}=M^{(s)} m^{-1} h_{p s}-\sum_{\tau=0}^{s-1} h_{p \tau}, \quad h_{j p s}^{(k)}=(-1)^{p} \frac{M_{j, s}}{2 i} \frac{\partial}{\partial \xi_{s}}+F_{j}^{(k)} \xi_{s}, \\
D_{-}^{(k)}=B \sum_{j \in C_{1}^{(k)}} e_{j}\left(q_{j 1}^{(k)}-q_{j 2}^{(k)}\right), \\
h_{p s}=(-1)^{p} \frac{M_{n, s}}{2 i} \frac{\partial}{\partial \xi_{s}}+2 e \xi_{s}, \quad M_{j, s}=\frac{m m_{j}}{M^{(s-1)} M^{(s)}}, \quad M^{(-1)} \equiv m, \\
F_{j}^{(k)}=B e_{j}+B m_{j} Q^{(k)}\left(M^{(k)}\right)^{-1}, \quad Q^{(s)}=Q-s e, \\
V^{(k)}=\frac{1}{2} \sum_{s, t=1 ; s \neq t}^{n} e_{s} e_{t}\left|q_{s}^{(k)}-q_{t}^{(k)}\right|^{-\gamma} .
\end{gathered}
$$


Отметим, что операторы $h_{j p s}^{(k)}$ при $j \geqslant 2$ не зависят от $j$ в силу тождественности электронов.

Пусть

$$
\mu^{(k+1)}=\inf H_{0}^{(k+1)}
$$

В разделе 4 будет доказана

Теорема 3.1. Для всех $k, k=1,2, \ldots, n-2, \sigma_{\mathrm{ess}}\left(H_{0}^{(k)}\right) \subseteq\left[\mu^{(k+1)},+\infty\right)^{2)}$. Ecлu $\mu^{(k+1)} \in \sigma_{\mathrm{p}}\left(H_{0}^{(k+1)}\right), m o$

$$
\inf H_{0}^{(k)}<\mu^{(k+1)}
$$

Из теоремы 3.1 элементарно следует выполнение условия (3.6). Действительно, по построению спектр оператора $H_{0}^{(n-1)}$ является чисто точечным, так как может содержать только точки, отвечающие орбитам Ландау исходной $n$-частичной системы. Значит, $\mu^{(n-1)} \in \sigma_{\mathrm{p}}\left(H_{0}^{(n-1)}\right)$. Тогда в силу неравенства $(3.15) \mu^{(n-2)} \in \sigma_{\mathrm{p}}\left(H_{0}^{(n-2)}\right)$. Если $n-1>2$, то, применяя теорему 3.1 при $k+1=n-3$, получаем, что $\mu^{(n-3)} \in \sigma_{\mathrm{p}}\left(H_{0}^{(n-3)}\right)$, и т.д. Таким образом последовательно мы придем к неравенству $\mu^{(1)}<\mu^{(2)}=\inf H_{0}^{(2)}$. Но $\mu^{(1)}=\mu$, так как $H_{0}^{(1)}=\widehat{H}_{0}\left(Z_{2}, 0\right)$. Поэтому неравенство $\mu^{(1)}<\mu^{(2)}$ - это неравенство (3.12), из которого и следует (3.6). Таким образом, теорему 2.2 можно считать доказанной, если верна теорема 3.1 .

\section{4. ДОКАЗАТЕЛЬСТВО ТЕОРЕМЫ 3.1}

4.1. Сначала в п. 4.1-4.3 мы приведем ту часть доказательства теоремы 3.1, которая нужна для обоих ее утверждений; непосредственные доказательства их даны соответственно в п. 4.4 и 4.5 .

Пусть $k$ фиксировано, $r=k+1$ и разбиение $Z_{r+1}=\left(C_{1}^{(r)}, \ldots, C_{r+1}^{(r)}\right)$ получено из $Z_{r}$ удалением из $C_{1}^{(k)}$ электрона с номером $(n-k)$, т.е. $C_{1}^{(r)}=(1,2, \ldots, n-r), C_{s}^{(r)}=n+$ $s-r-1, s=2, \ldots, r+1$. Положим

$$
F=H_{0}^{(k)}-\sum_{s=1}^{n-r} e_{s} e\left|q_{s}^{(k)}-q_{n-k}^{(k)}\right|^{-\gamma}
$$

Перейдем в операторе $F$ к новым координатам аналогично тому, как мы это делали в п. 2.2 с той, однако, разницей, что роль исходной системы $Z_{1}$ играет кластер $C_{1}^{(k)}$, роль кластеров $C_{1}, C_{2}$ - кластеры $C_{1}^{(r)}, C_{2}^{(r)}$, и относительные координаты в кластере $C_{2}^{(r)}$ не вводятся, так как он одночастичный. Итак, пусть

$$
\begin{gathered}
\alpha_{1}^{\prime}=\left(\alpha_{11}^{\prime}, \alpha_{12}^{\prime}, \alpha_{13}^{\prime}\right)=\sum_{s=1}^{n-r} \frac{m_{s} q_{s}^{(k)}}{M^{(r)}}, \quad \alpha_{2}^{\prime}=q_{n-k}^{(k)}, \quad q_{j}^{\prime}=q_{j}^{(k)}-\alpha_{1}^{\prime}, \quad j \in C_{1}^{(r)}, \\
\xi^{\prime}=\xi_{r}=\frac{\alpha_{11}^{\prime}-\alpha_{12}^{\prime}}{2}, \quad \eta^{\prime}=\eta_{r}=\frac{\alpha_{11}^{\prime}+\alpha_{12}^{\prime}}{2}, \quad \zeta^{\prime}=\zeta_{r}=\alpha_{13}^{\prime}-\alpha_{23}^{\prime} .
\end{gathered}
$$

\footnotetext{
2) Можно доказать, что здесь вместо включения справедливо равенство, но для данной работы это не существенно.
} 
Так как $M^{(r)} \alpha_{1}^{\prime}+m \alpha_{2}^{\prime}=\sum_{j \in C_{1}^{(k)}} m_{j} q_{j}^{(k)}=0$, то $\alpha_{2}^{\prime}=-M^{(r)} m^{-1} \alpha_{1}^{\prime}$. С учетом этого старые переменные $q_{j p}^{(k)}, j=1, \ldots, n-k, p=1,2,3$, и новые $q_{j p}^{(r)} \equiv q_{j p}^{\prime}, j=1, \ldots, n-r$, $p=1,2,3, \xi^{\prime}, \eta^{\prime}, \zeta^{\prime}$ связаны соотношениями

$$
\begin{gathered}
q_{j p}^{(k)}=q_{j p}^{\prime}+\left(\eta^{\prime}-(-1)^{p} \xi^{\prime}\right), \quad j=1,2, \ldots, n-r, \\
q_{n-k, p}^{(k)}=M^{(r)} m^{-1}\left((-1)^{p} \xi^{\prime}-\eta^{\prime}\right), \quad p=1,2, \\
q_{j 3}^{(k)}=q_{j 3}^{\prime}+\frac{m \zeta^{\prime}}{M^{(k)}}, \quad j=1,2, \ldots, n-r, \quad q_{n-k, 3}^{(k)}=\frac{m \zeta^{\prime}}{M^{(k)}} .
\end{gathered}
$$

Отметим, что переменные $\xi^{(s)}=\left(\xi_{0}, \ldots, \xi_{s}\right), s=k$, в замене не участвуют и остаются без изменений. В силу (4.1)-(4.3) оператор $F$ можно записать в виде

$$
F=F_{0}+F_{\mathrm{c}}
$$

где

$$
\begin{aligned}
& F_{0}= \sum_{p=1}^{2}\left\{\sum _ { j = 1 } ^ { n - r } m _ { j } ^ { - 1 } \left[B_{j p}^{(r)}-(-1)^{p} B e_{j} q_{j \bar{p}}^{\prime}-m_{j}\left(2 M^{(k)}\right)^{-1} D_{-}^{(r)}+\right.\right. \\
&+\frac{M_{j, r}}{2 i}\left(\frac{\partial}{\partial \eta^{\prime}}-(-1)^{p} \frac{\partial}{\partial \xi^{\prime}}\right)-e_{j}\left(\xi^{\prime}+(-1)^{p} \eta^{\prime}\right) B- \\
&\left.-m_{j} \xi^{\prime}\left(m Q^{(r)}-e M^{(r)}\right) \frac{B}{m M^{(k)}}-\sum_{s=0}^{k} h_{j p s}^{(k)}\right]^{2}+ \\
&+\left[\frac{m}{2 M^{(k)} i}\left((-1)^{p} \frac{\partial}{\partial \xi^{\prime}}-\frac{\partial}{\partial \eta^{\prime}}\right)+e M^{(r)} B\left(\xi^{\prime}+\eta^{\prime}(-1)^{p}\right) m^{-1}-\frac{m D_{-}^{(r)}}{2 M^{(k)}}-\right. \\
&\left.\left.-\xi^{\prime} B \frac{Q^{(r)} m-e M^{(r)}}{M^{(k)}}-\sum_{s=1}^{k} h_{n p s}^{(k)}\right]^{2}\right\}+T_{c \perp}^{(r)}+T_{03}^{(r)}+V^{(r)} ; \\
& F_{c}=-\frac{M^{(k)}}{m M^{(r)}} \frac{d^{2}}{d \zeta^{\prime 2}} .
\end{aligned}
$$

4.2. Подберем теперь линейную комбинацию $\omega_{k}$ переменных $q_{t p}^{(r)}, \xi_{s}$ так, чтобы оператор

$$
\widetilde{F}_{0}:=e^{i \omega_{k} \eta^{\prime}} F_{0} e^{-i \omega_{k} \eta^{\prime}}
$$

не содержал членов вида $c \eta^{\prime}$, а потом положим

$$
H_{0}^{(r)}\left(\lambda^{\prime}\right)=\int \widetilde{F}_{0} e^{-i \eta^{\prime} \lambda^{\prime}} d \eta^{\prime}
$$

где $\lambda^{\prime} \in R^{1}$ и оператор (4.6) рассматривается в пространстве $R^{(3 n-2 r-1)}=\left\{q^{(r)}\right.$, $\left.\xi_{0}, \ldots, \xi_{r}, \lambda^{\prime}\right\}$. Весьма громоздкие вычисления показывают, что при

$$
\omega_{k}=D_{-}^{(r)}+2 \sum_{s=0}^{k} \xi_{s}\left(Q^{(r)} m-M^{(r)} e\right)+2 \xi^{\prime} m^{-2}\left(Q^{(r)} m^{2}+e\left(M^{(r)}\right)^{2}\right)
$$


оператор $\widetilde{F}_{0}$ действительно не будет содержать членов $c \eta^{\prime}$, а оператор (4.6) будет иметь вид

$$
\begin{aligned}
H_{0}^{(r)}\left(\lambda^{\prime}\right)= & \sum_{j=1}^{n-r} \sum_{p=1}^{2} m_{j}^{-1}\left[A_{j p}^{(r)}-\sum_{s=0}^{r} h_{j p s}^{(r)}+\frac{1}{2} M_{j, r} \lambda^{\prime}\right]^{2}+ \\
& +m^{-1} \sum_{s=1}^{r} \sum_{p=1}^{2}\left(\ell_{p s}-\delta_{s, r} \frac{m \lambda^{\prime}}{2 M^{(k)}}\right)^{2}+T_{03}^{(r)} .
\end{aligned}
$$

По построению $F_{0}$ унитарно эквивалентен $H_{0}^{(r)}\left(\lambda^{\prime}\right)$, если эти операторы рассматривать соответственно в пространствах переменных $q^{(r)}, \xi^{(r)}, \eta^{\prime}$ и $q^{(r)}, \xi^{(r)}, \lambda^{\prime}$. Если оператор $H_{0}^{(r)}\left(\lambda^{\prime}\right)$ будет рассматриваться при фиксированном $\lambda^{\prime}$, т.е. в пространстве $R^{(3 n-2 r-2)}=\left\{q^{(r)}, \xi^{(r)}\right\}$, то мы будем обозначать его через $\widehat{H}_{0}^{(r)}\left(\lambda^{\prime}\right)$.

4.3. При $r=1$ операторы $H_{0}^{(r)}\left(\lambda^{\prime}\right)$ и $\widehat{H}_{0}^{(r)}\left(\lambda^{\prime}\right)$ совпадают соответственно с операторами $H_{0}\left(Z_{2}, \lambda^{\prime}\right)$ и $\widehat{H}_{0}\left(Z_{2}, \lambda^{\prime}\right)$ при $Z_{2}=\left\{\left(Z_{1} \backslash n\right),(n)\right\}$ (см. (2.6)) и, следовательно, их свойства даются теоремой 2.3 из работы [1]. Свойства операторов $H_{0}^{(r)}\left(\lambda^{\prime}\right)$ и $\widehat{H}_{0}^{(r)}\left(\lambda^{\prime}\right)$ при $r \geqslant 2$ аналогичны, а именно спектр $\sigma\left(\widehat{H}_{0}^{(r)}\left(\lambda^{\prime}\right)\right)$ не зависит от $\lambda^{\prime}$ и спектр $\sigma\left(H_{0}^{(r)}\left(\lambda^{\prime}\right)\right)$ совпадает со спектром $\sigma\left(\widehat{H}_{0}^{(r)}(0)\right)$, отличаясь от него лишь дополнительным бесконечным вырождением.

Доказательство этих утверждений проведем одновременно. Пусть $b_{t}, t=1,2, \ldots$ $\ldots, n-r$ и $\gamma_{\tau}, \tau=0,1, \ldots, r,-$ произвольные числа, $\omega^{\prime}=\sum_{t=1}^{n-r} b_{t}\left(q_{t 1}^{\prime}+q_{t 2}^{\prime}\right)$. Сделаем калибровочное преобразование

$$
e^{i \omega^{\prime} \lambda^{\prime}} H_{0}^{(r)}\left(\lambda^{\prime}\right) e^{-i \omega^{\prime} \lambda^{\prime}}
$$

и затем в операторе (4.8) заменим $\xi_{\tau}$ на $\xi_{\tau}+\gamma_{\tau} \lambda^{\prime}$. В полученном после этого операторе по сравнению с $H_{0}^{(r)}\left(\lambda^{\prime}\right)$ изменятся только члены с $\lambda^{\prime}$. Обозначим через $d_{j}$ и $d_{s}^{\prime}$ коэфффициенты перед $\lambda^{\prime}$, которые получатся после преобразования (4.8) соответственно в $j$-м слагаемом первой суммы и в $s$-м слагаемом второй суммы равенства (4.7). Вычисления показывают, что

$$
\begin{aligned}
& d_{j}=- \sum_{s=1}^{n-r}\left(\delta_{s j}-m_{j} M^{(r)^{-1}}\right) b_{s}-\sum_{\tau=0}^{r}\left(e_{j} M^{(r)}+Q^{(r)} m_{j}\right) B M^{(r)^{-1}} \gamma_{\tau}+\frac{1}{2} M_{j, r}, \\
& j=1,2, \ldots, n-r, \\
& d_{s}^{\prime}=-2 \sum_{\tau=0}^{s-1} B e \gamma_{\tau}+2 e B m^{-1} M^{(s)} \gamma_{s}-\frac{1}{2} m M^{(k)^{-1}} \delta_{s, r}, \quad s=1,2, \ldots, r .
\end{aligned}
$$

Докажем, что при некоторых $b_{t}$ и $\gamma_{\tau}$

$$
d_{j}=0, \quad j=1, \ldots, n-r ; \quad d_{s}^{\prime}=0, \quad s=1, \ldots, r .
$$


Полагая в (4.10) последовательно $s=1,2, \ldots, r-1$, получаем $\gamma_{1}=\beta_{1} \gamma_{0}, \gamma_{2}=\beta_{2} \gamma_{0}$, $\ldots, \gamma_{r-1}=\beta_{r-1} \gamma_{0}$ и при $s=r$

$$
M^{(r)} m^{-1} \gamma_{r}-\beta \gamma_{0}=\frac{m}{8 M^{(k)}} e B
$$

где $\beta_{s}>0$ - известные числа, $\beta=\sum_{s=1}^{r-1} \beta_{s}$. Далее, суммируя (4.10) по $j$, получим

$$
-2 B Q^{(r)} \sum_{\tau=0}^{r} \gamma_{\tau}+\frac{m}{2 M^{(k)}}=0,
$$

T.e.

$$
\gamma_{r}+\beta \gamma_{0}=\frac{m}{4 Q^{(r)} M^{(k)} B} .
$$

Из (4.11), (4.13) находим $\gamma_{0}, \gamma_{r}$ и затем $\gamma_{s}=\beta_{s} \gamma_{0}, s=1,2, \ldots, r-1$.

Таким образом мы нашли числа $\gamma_{s}, s=0, \ldots, r$, обеспечивающие справедливость $d_{s}^{\prime}=0$ и (4.12). Решение $d_{j}=0$ ишем в виде $b_{s}=b e_{s}+\tilde{b} m_{s}$. Подставляя это выражение в (4.10) и приравнивая нулю коэффициенты при $e_{j}$ и $m_{j}$ при каждом $j$, видим, что в силу (4.12) $b=M /\left(4 M^{(k)} Q^{(r)}\right)$, и $\tilde{b}$ можно взять равным нулю. Из сказанного вытекает, что после калибровочного преобразования (4.8) и замены $\xi_{\tau} \rightarrow \xi_{\tau}+\gamma_{\tau} \lambda^{\prime}$ с найденными $\gamma_{\tau}$ и $b_{s}$ мы получим оператор, не содержаший $\lambda^{\prime}$, т.е. $H_{0}^{(r)}(0)$. По построению $H_{0}^{(r)}(0)$ унитарно эквивалентен $H_{0}^{(r)}\left(\lambda^{\prime}\right)$ в $R^{(3 n-2 r-1)}$, и так как $H_{0}^{(r)}(0)$ не зависит от $\lambda^{\prime}$, то утверждения п. 4.3 доказаны.

4.4. Докажем второе из утверждений теоремы 3.1. Пусть $\mu^{(r)} \in \sigma_{\mathrm{p}}\left(H_{0}^{(r)}\right)$. Вследствие второго утверждения п. 4.3 и поскольку $H_{0}^{(r)}=\widehat{H}_{0}^{(r)}(0), \mu^{(r)}$ - собственное значение оператора $F_{0}$, унитарно эквивалентного $H_{0}^{(r)}\left(\lambda^{\prime}\right)$ и $H_{0}^{(r)}(0)$. В силу $(4.1)$ и $(4.4)$

$$
H_{0}^{(k)}=F_{0}+F_{\mathrm{c}}+\frac{1}{2} \sum_{s=1}^{n-r} e_{s} e\left|q_{s}^{(k)}-q_{n-k}^{(k)}\right|^{-\gamma} .
$$

Пусть $u$ - нормированная собственная функция оператора $F_{0}$, отвечающая числу $\mu^{(r)}$, $g\left(\zeta^{\prime}\right) \in C_{0}^{2}, \quad \operatorname{supp} g\left(\zeta^{\prime}\right) \in[1,2], \quad g_{\alpha}=g\left(\alpha \zeta^{\prime}\right) \alpha^{1 / 2}, u_{\alpha}=u g_{\alpha}, \quad R^{\prime}=\left\{\zeta^{\prime}\right\}$. Тогда при $\|g\|=1$

$$
\left(H_{0}^{(k)} u_{\alpha}, u_{\alpha}\right)=\left(F_{0} u, u\right)+\left(F_{c} g_{\alpha}, g_{\alpha}\right)_{R^{\prime}}+\frac{1}{2} \sum_{s=1}^{n-r} e_{s} e\left|\widetilde{P}_{s}\right|^{-\gamma}
$$

где $\left(F_{0} u, u\right)=\mu^{(r)}, \quad\left(F_{\mathrm{c}} g_{\alpha}, g_{\alpha}\right)=\alpha^{2}\left(F_{\mathrm{c}} g_{1}, g_{1}\right), \widetilde{P}_{s}=\left(\widetilde{P}_{s 1}, \widetilde{P}_{s 2}, \widetilde{P}_{s 3}\right)=q_{s}^{(k)}-q_{n-k}^{(k)}=$ $\left(q_{s 1}^{(r)}+m^{-1} M^{(k)}\left(\xi_{r}+\eta^{\prime}\right), q_{s 2}^{(r)}+m^{-1} M^{(k)}\left(\eta^{\prime}-\xi_{r}\right), q_{s 3}^{(r)}+\zeta^{\prime}\right)$. Действуя так же, как в п. 3.3, убеждаемся, что при малых $\alpha$

$$
\left(H_{0}^{(k)} u_{\alpha}, u_{\alpha}\right) \leqslant \mu^{(r)}+\alpha^{2} a_{1}+\alpha^{\gamma} Q^{(n-r)} e a_{2},
$$

где $a_{i}>0$ - некоторые числа, $Q^{(n-k)} e<0$. Отсюда при малых $\alpha$ имеем

$$
\left(H_{0}^{(k)} u_{\alpha}, u_{\alpha}\right)<\mu^{(r)},
$$

т.е. второе утверждение теоремы 3.1 доказано. 
4.5. Докажем первое утверждение теоремы 3.1. Пусть $q^{(k)}=\left(q_{1}^{(k)}, \ldots, q_{n-k}^{(k)}\right), \xi^{(k)}=$ $\left(\xi_{0}, \ldots, \xi_{k}\right), R=\left\{q^{(k)}, \xi^{(k)}\right\}$. Так как для любого $\nu \in \sigma_{\mathrm{ess}}\left(H_{0}^{(k)}\right)$ сушествует последовательность $g_{m}=g_{m}\left(q^{(k)}, \xi^{(k)}\right) \in C_{0}^{2}$, такая, что

$$
\lim _{m \rightarrow \infty}\left(H_{0}^{(k)} g_{m}, g_{m}\right)=\nu, \quad g_{m} \underset{m \rightarrow \infty}{\rightarrow} 0 \quad \text { в } \quad \mathcal{L}_{2}(R), \quad\left\|g_{m}\right\|=1
$$

то будет достаточно доказать, что неравенство

$$
\underline{\lim }\left(H_{0}^{(k)} g_{m}, g_{m}\right) \geqslant \mu^{(k+1)}
$$

верно для любой последовательности $g_{m}$ со свойствами (4.14).

Пусть $\Omega_{b}=\left\{\left(q^{(k)}, \xi^{(k)}\right)|| q^{(k)} \mid \leqslant b\right\}$. Для получения (4.15) решающую роль будет играть оценка

$$
\lim _{m \rightarrow \infty}\left\|g_{m}\right\|_{\Omega_{b}}=0
$$

которую мы докажем при любом $b>0$.

4.6. Перестроим оператор $T_{0 \perp}^{(k)}$ из $(3.14)$, используя тождество $(3.12)$ из работы [1] с

$$
f_{j p}=\left(A_{j p}^{(k)}-\sum_{s=0}^{k} h_{j p s}^{(k)}\right) m_{j}^{-1}, \quad n_{0}=n-k, \quad \mathcal{M}=M^{(k)} .
$$

Тогда (см. [1], п. 3.5) мы получим, что

$$
T_{0 \perp}^{(k)}=(f, f)_{0}=\sum_{j=1}^{n-k} \sum_{p=1}^{2} m_{j} f_{j p}^{2}=\sum_{j=1}^{n-k} \sum_{p=1}^{2}\left(f_{j p}-f_{\mathrm{c} p}\right)^{2} m_{j}+M^{(k)} \sum_{p=1}^{2} f_{\mathrm{c} p}^{2}
$$

где

$$
\begin{aligned}
f_{\mathrm{c} p}=\sum_{j=1}^{n-k} m_{j} \frac{f_{j p}}{M^{(k)}} & =\left((-1)^{p+1} \sum_{j=1}^{n-k} e_{j} q_{j \bar{p}}^{(k)}-\frac{1}{2} D_{-}^{(k)}-\sum_{s=0}^{k} h_{\mathrm{c} p s}^{(k)}\right)\left(M^{(k)}\right)^{-1}, \\
h_{\mathrm{c} p s}^{(k)} & =\frac{m M^{(k)}}{2 M^{(s-1)} M^{(s)} i}(-1)^{p} \frac{\partial}{\partial \xi_{s}}+2 B Q^{(k)} \xi_{s} .
\end{aligned}
$$

Так как оператор $H_{0}^{(k)}-(1 / 2)\left(T_{0 \perp}^{(k)}+T_{\mathrm{c} \perp}^{(k)}+T_{03}^{(k)}\right)$ ограничен снизу, а операторы $f_{j p}$, $f_{\mathrm{c} p}, f_{s p}, B_{j 3}^{(k)}$ симметричны, то в силу первого из условий $(4.14)$ и $(4.17)$

$$
\begin{aligned}
L:= & \sup _{m}\left\{\sum_{j=1}^{n-k}\left[\left\|B_{j 3}^{(k)} g_{m}\right\|_{\Omega}^{2}+\sum_{p=1}^{2}\left\|\left(f_{j p}-f_{c p}\right) g_{m}\right\|_{\Omega}^{2}\right]+\right. \\
& \left.+\sum_{p=1}^{2}\left[\left\|f_{c p} g_{m}\right\|_{\Omega}^{2} M^{(k)}+\sum_{s=1}^{k}\left\|\ell_{p s} g_{m}\right\|_{\Omega}^{2}\right]\right\}<+\infty
\end{aligned}
$$


для любой области $\Omega \subset R$. Раскрывая в (4.18) выражение в фигурных скобках и приводя подобные члены, видим, что в полученном результате произведения $\xi_{t} \partial \bar{g}_{m} / \partial \xi_{s}$, $\xi_{t} \partial g_{m} / \partial \xi_{s}$ отсутствуют, а выражения, содержашие произведения $\xi_{s} \xi_{t}\left|g_{m}\right|^{2}, \quad s, t=$ $0,1, \ldots, k$, и произведения $\left(\partial \bar{g}_{m} / \partial \xi_{s}\right)\left(\partial g_{m} / \partial \xi_{t}\right), s, t=0,1, \ldots, k$, являются положительно определенными квадратичными формами соответственно по $\xi_{s}\left|g_{m}\right|, \quad s=$ $0,1, \ldots, k$, и по $\left|\partial g_{m} / \partial \xi_{s}\right|, s=0,1, \ldots, k$. Поэтому из (4.18) следует, что для некоторого $\delta_{0}>0$

$$
L \geqslant 2 \delta_{0} \sum_{s=0}^{k}\left(\left\|\xi_{s} g_{m}\right\|_{\Omega}^{2}+\left\|\frac{\partial g_{m}}{\partial \xi_{s}}\right\|_{\Omega}^{2}\right)+\left(\Phi g_{m}, g_{m}\right)_{\Omega},
$$

где $\Phi$ - вешественная часть линейной комбинации членов $q_{j p}^{(k)} q_{j_{1} p_{1}}^{(k)},(1 / i) q_{j p}^{(k)} d g_{m} / d \xi_{s}$, $q_{j p}^{(k)} \xi_{t}, j, j_{1}=1,2, \ldots, n-k, s=1,2, \ldots, k, p, p_{1}=1,2$. Пусть теперь в (4.19) $\Omega=\Omega_{b}$. Так как $\left|q^{(k)}\right| \leqslant b$ в $\Omega_{b}$, то

$$
L \geqslant \delta_{0} \sum_{s=0}^{k}\left(\left\|\xi_{s} g_{m}\right\|_{\Omega_{b}}^{2}+\left\|\frac{\partial g_{m}}{\partial \xi_{s}}\right\|_{\Omega_{b}}^{2}\right)-C
$$

где $C$ зависит от $b$, но не от $m$. Пусть $\Omega_{b, N}=\left\{\left(q^{(k)}, \xi^{(k)}\right)|| q^{(k)}|\leqslant b,| \xi^{(k)} \mid \leqslant N\right\}$. Полагая в (4.18) $\Omega=\Omega_{b, N}$ и учитывая (4.20), получаем, что $\sup _{m}\left\|B_{j p}^{(k)} g_{m}\right\|_{\Omega_{b, N}}<+\infty, j=$ $1,2, \ldots, n-k, p=1,2,3$. Это неравенство вместе с (4.20) позволяет применить к последовательности $g_{m}$ в области $\Omega_{b, N}$ теорему вложения Соболева. Используя ее и (4.14), заключаем, что при любых фиксированных $b, N$ выполняется $\lim _{m \rightarrow \infty}\left\|g_{m}\right\|_{\Omega_{b, N}}=0$. Поэтому и в силу $(4.20)$ при любом $N>0$

$$
L \geqslant \delta_{0} N \varlimsup_{m \rightarrow \infty}\left\|g_{m}\right\|_{\Omega_{b}}-C
$$

что возможно лишь при выполнении (4.16), т.е. утверждение (4.16) доказано.

4.7. Пусть $\bar{Z}_{1}=C_{1}^{(k)}$ и $\bar{Z}_{\alpha}=\left(\bar{C}_{1}, \ldots, \bar{C}_{\alpha}\right)$ - разбиение кластера $C_{1}^{(k)}=\bar{Z}_{1}$ на $\alpha$ не пересекающихся кластеров $\bar{C}_{j}$,

$$
H_{0}^{(k)}\left(\bar{Z}_{\alpha}\right)=T_{0}+\sum_{j=1}^{\alpha} V\left[\bar{C}_{j}\right], \quad \nu_{0}=\min _{\bar{Z}_{\alpha}, \alpha \geqslant 2} \inf H_{0}^{(k)}\left(\bar{Z}_{\alpha}\right)
$$

Так как $V_{s t}>0$ при $s, t \neq 1$, то $\nu_{0}=\inf H_{0}^{(k)}\left(\bar{Z}_{2}\right)$, где $\bar{Z}_{2}=\left\{\left(\bar{Z}_{1} \backslash(n-k-1)\right),(n-k-1)\right\}$. Для произвольных $b(1)>a(1)>1$ определим функции $u_{1}(\tau), v_{1}(\tau) \in C^{2}\left(R^{1}\right): u_{1}(\tau)=1$ при $\tau \in[0, a(1)], u_{1}(\tau) \in(0,1)$ при $\tau \in(a(1), b(1)), u_{1}(\tau) \equiv 0$ при $\tau \geqslant b(1), v_{1}^{2}(\tau)+$ $u_{1}^{2}(\tau)=1$. Положим $\left|q^{(k)}\right|_{0}=\sum_{j=1}^{n-k}\left|q_{j}^{(k)}\right|^{2} m_{j}, \tau_{\bar{Z}_{1}}=\left|q^{(k)}\right|_{0}, \quad u_{\bar{Z}_{1}}=u_{1}\left(\tau_{\bar{Z}_{1}}\right), v_{\bar{Z}_{1}}=$ $v_{1}\left(\tau_{\bar{Z}_{1}}\right)$. Пусть число $m$ велико и фиксировано, $\psi_{0, \bar{Z}_{1}}=g_{m} u_{\bar{z}_{1}}, \hat{\psi}_{1}=g_{m} v_{\bar{Z}_{1}}$. Тогда для любого $\varepsilon>0$ можно выбрать числа $a(1), b(1)$ и функцию $u_{1}(\tau)$ такие, что для всех $m$

$$
\left(H_{0}^{(k)} g_{m}, g_{m}\right) \geqslant\left(H_{0}^{(k)} \psi_{0, \bar{Z}_{1}}, \psi_{0, \bar{Z}_{1}}\right)+\left(H_{0}^{(k)} \hat{\psi}_{1}, \hat{\psi}_{1}\right)-\varepsilon
$$


Так как $\hat{\psi}_{1} \equiv 0$ при $\left|q^{(k)}\right|_{0} \leqslant a(1)$ и $\sum_{j=1}^{n-k} m_{j} q_{j}^{(k)}=0$, то оценка снизу второго слагаемого в (4.22) проводится стандартным образом: фиксируем $\xi^{(k)}$, разбиваем пространство переменных $q^{(k)}$ на области, отвечаюшие всевозможным распадениям системы $\bar{Z}_{1}=C_{1}^{(k)}$ и оцениваем квадратичную форму оператора $H_{0}^{(k)}$ по этим областям при большом $a(1)$ (для чего можно следовать работе [4], с. $420-421$, взяв там $\bar{Z}_{1}$ вместо $Z_{1}$ ), а потом интегрируем по $\xi^{(k)}$. В результате получим

$$
\left(H_{0}^{(k)} \hat{\psi}_{1}, \hat{\psi}_{1}\right) \geqslant \nu_{0}\left\|\hat{\psi}_{1}\right\|^{2}-\varepsilon,
$$

где $\varepsilon$ можно взять сколь угодно малым, если $a(1)$ велико. Далее, $\left\|\psi_{0}, \bar{Z}_{1}\right\|^{2} \leqslant\left\|g_{m}\right\|_{\Omega_{b}}^{2}$, и в силу (4.16) при больших $m$ вьполняется $\mu\left\|\psi_{0, \bar{Z}_{1}}\right\|^{2} \geqslant \nu_{0}\left\|\psi_{0, \bar{Z}_{1}}\right\|^{2}-\varepsilon$. Поэтому при больших $m$

$$
\left(H_{0}^{(k)} \psi_{0, \bar{Z}_{1}}, \psi_{0, \bar{Z}_{1}}\right) \geqslant \nu_{0}\left\|\psi_{0, \bar{Z}_{1}}\right\|^{2} \geqslant \nu_{0}\left\|\psi_{0, \bar{Z}_{1}}\right\|^{2}-\varepsilon .
$$

Из (4.22)-(4.24) следует, что при больших $m$

$$
\left(H_{0}^{(k)} g_{m}, g_{m}\right) \geqslant \nu_{0}\left\|g_{m}\right\|^{2}-3 \varepsilon .
$$

Но $H_{0}^{(k)}\left(\bar{Z}_{2}\right)=F($ см. п. 4.1), поэтому и вследствие (4.4)-(4.6) и утверждений п. 4.3

$$
\nu_{0}=\inf H_{0}^{(k)}\left(\bar{Z}_{2}\right)=\mu^{(k+1)} .
$$

Значит, (4.25) доказывает первое утверждение теоремы 3.1.

\section{Список литературы}

[1] Г. М. Жислин. ТМФ. 2002. Т. 133. № 1. С. 87-102.

[2] J. E. Avron, J. W. Herbst, B. Simon. Ann. Phys. 1978. V. 114. P. 431-451.

[3] Г. М. Жислин. Докл. РАН. 2001. Т. 377. № 3. С. 301-304.

[4] С. А. Вугальтер, Г. М. Жислин. ТМФ. 1997. Т. 113. № 3. С. 413-431.

Поступила в редакцию 18.I.2002 г. 